\title{
Características de los aspirantes a la Escuela Normal en México. ¿̇En qué condiciones inicia su formación el profesorado de primaria? ${ }^{1}$
}

\author{
Characteristics of Applicants to the Normal School in Mexico: \\ Under what Conditions Does the Elementary School Teachers Start their \\ Teacher Training?
}

Jihan García-Poyato Falcón y Graciela Cordero Arroyo²

\section{Resumen}

En México la formación de profesores de preescolar y primaria se lleva a cabo tradicionalmente en las Escuelas Normales (EN), espacio específico asignado por la autoridad educativa federal para la formación inicial docente. En este estudio cuantitativo se buscó identificar las diferencias entre las características de 345 aspirantes a la Licenciatura en Educación Primaria en seis EN públicas de Baja California (generación 2018-2022), entidad ubicada al noroeste del país. Dichas características fueron recuperadas a través de una encuesta y la información se analizó con distintas pruebas de estadística descriptiva e inferencial. En los resultados se presentan las características de los aspirantes (laborales, socioeconómicas, familiares y académicas), así como la comparación realizada para puntualizar las diferencias significativas entre los grupos de aspirantes por EN y por su ubicación en zona urbana o rural. Se encontró que los aspirantes de la zona rural se encuentran en desventaja en los tres ámbitos analizados: socioeconómico, familiar y académico. La información recuperada es un insumo imprescindible para el diseño de políticas educativas, de carácter institucional, estatal y federal, que permitan el acompañamiento de los aspirantes una vez que ingresen a la EN, como la implementación de programas de becas y tutorías individualizadas.

\section{Palabras clave}

Escuela de formación de profesores, docente de escuela primaria, formación de docentes de primaria, candidato, características.

\section{Abstract}

In Mexico the teacher education for preschool and primary teachers is traditionally in the Normal Schools (NS), a specific space assigned by the federal educational authority for initial teacher training. In this quantitative study, we sought to identify the differences between the characteristics of 345 applicants for the Bachelor of Arts in Primary Education in six public NS of Baja California (generation 2018-2022), located in the northwest of the country. Those characteristics were collected with a survey and the information was analyzed with different descriptive and inferential statistics tests. The results show the characteristics of the participants (labor, socioeconomic, family and academic), as well as the comparison made to point out the significant differences between the groups of applicants to each NS and their location in an urban or rural area. The information retrieved is an essential input for the design of educational policies, in the institutional, state and federal level, that allows the accompaniment of applicants once they enter the NS, such as the implementation of scholarship programs and individualized tutoring.

\section{Keywords}

Teacher training school, elementary school teacher, training of elementary school teacher, candidate, characteristics.

\section{Cómo citar/Citation}

García-Poyato Falcón, Jihan; Cordero Arroyo, Graciela (2019). Características de los aspirantes a la Escuela Normal en México. ¿En qué condiciones inicia su formación el profesorado de primaria? Revista de Sociología de la Educación-RASE, 12 (2), 157-175. http://dx.doi. org/10.7203/RASE.12.2.14659.

\footnotetext{
1 Este artículo forma parte del proyecto TO-INN From Tradition to Innovation in Teacher Training Institutions financiado por la Unión Europea a través del programa Erasmus, Acción K2 Capacity Building, (573685-EPP-2016-1-ES-EPPKA2-CBHE-JP) https://www.toinn.org.

2 Jihan García-Poyato Falcón, Benemérita Escuela Normal Estatal, jihan.gpoyato@gmail.com; Graciela Cordero Arroyo, Universidad Autónoma de Baja California, cordero@uabc.edu.mx.
} 


\section{Introducción}

La figura del profesor tiene un gran impacto en la sociedad, más aún de los profesores dedicados a la enseñanza elemental debido al cercano rol de su profesión en la formación de los niños. De esta manera, «el docente como agente intelectual y social tiene la posibilidad de tener una participación activa en la formación de actores sociales que busquen el desarrollo de sociedades justas y humanizadas» (Loubet-Orozco, 2018: 18).

En México las Escuelas Normales (EN) son el espacio específico asignado por la autoridad educativa federal para la formación inicial del profesorado de preescolar y primaria. Corresponde al nivel de Educación Superior (ES) y sus programas consisten en esquemas de formación que integran aspectos disciplinares y pedagógicos, con espacios simultáneos de inmersión en contextos reales de trabajo docente (Fortoul, Güemes, Martel y Reyes, 2013).

Se considera relevante enfocar esfuerzos de investigación en conocer a los candidatos que pretenden ingresar a sus aulas para formarse como docentes, quienes deben concluir el bachillerato como requisito académico previo a su ingreso.

A partir de la revisión de la literatura sobre el tema es posible afirmar que en el siglo xxi ha incrementado la producción académica de la investigación que tiene a los estudiantes como centro de su interés, sin embargo, pocos estudios se enfocan en las características de los aspirantes, es decir, en su etapa previa al ingreso a la ES.

En la Tabla I se presenta una síntesis de 10 estudios seleccionados, publicados en revistas arbitradas y memorias de congresos; de cada uno se recuperó objetivo, metodología y resultados principales. Se presentan con la intención de plantear de forma somera algunas de las aproximaciones realizadas en torno al objeto de estudio. Los criterios de búsqueda utilizados fueron: publicación en los últimos cinco años, escritos en inglés o español, acceso gratuito al texto completo, palabras clave como aspirante, candidato, ES, universidad, formación docente. 
Características de los aspirantes a la Escuela Normal en México. ¿̇En qué condiciones inicia su formación el profesorado de primaria?

Tabla I. Síntesis de la revisión de estudios previos

\begin{tabular}{|c|c|c|c|c|c|}
\hline AÑO & PAís & AUTOR (ES) & OBJETIVO DE LA INVESTIGACIÓN & METODOLOGÍA & RESULTADOS PRINCIPALES \\
\hline 2019 & Canadá & Litalien, Gillet, Gagné, Ratelle y Morin & $\begin{array}{l}\text { Examinar el perfil de estudiantes universitarios por el tipo de regulación } \\
\text { de comportamiento. }\end{array}$ & Cuantitativa, encuesta a 1072 estudiantes universitarios. & $\begin{array}{l}\text { Presentan cinco perfiles motivacionales conformados por caracteristicas } \\
\text { demográficas y académicas particulares, con efectos en su comporta- } \\
\text { miento durante sus estudios profesionales. }\end{array}$ \\
\hline 2019 & México & Jaimes, Reynoso y Ferrer & $\begin{array}{l}\text { Examinar el proceso de clasificación y selección de las aspirantes a } \\
\text { ingresar a una EN rural y su relación con sus características regionales. }\end{array}$ & $\begin{array}{l}\text { Cuantitativa, de tipo exploratorio-descriptivo, encuesta a } 428 \text { aspirantes } \\
\text { a la EN de los ciclos escolares 2017-2018 y } 2018-2019 \text {. }\end{array}$ & $\begin{array}{l}\text { Las aspirantes provienen de ocho estados cercanos. Sin importar } \\
\text { su región de origen, la mayoría presenta rezago en el desarrollo de } \\
\text { sus habilidades académicas y es una limitante en el logro de sus } \\
\text { competencicias profesesionales. }\end{array}$ \\
\hline 2018 & España & Alfonzo, Cáceres y Santana & $\begin{array}{l}\text { Enfatizar el análisis de las necesidades de los estudiantes para } \\
\text { desarrollar lazos interculturales. }\end{array}$ & $\begin{array}{l}\text { Cuantitativa, encuesta a } 60 \text { estudiantes no españoles participantes del } \\
\text { Proyecto Erasmus. }\end{array}$ & $\begin{array}{l}\text { Las correlaciones más altas se encontraron entre las variables movili- } \\
\text { dad, nivel académico y la edad de los participantes. }\end{array}$ \\
\hline 2018 & México & Hernández & $\begin{array}{l}\text { Determinar el perfil sociodemográfico y académico a partir del nivel de } \\
\text { autoeficacia académica. }\end{array}$ & Cuantitativa, encuesta a 182 estudiantes universitarios. & $\begin{array}{l}\text { Las variables con diferencia estadísticamente significativa son: contar } \\
\text { con una beca, el agrado de la carrera, dificultades económicas y deseos } \\
\text { de estudiar un posgrado. El perfil promedio de los participantes en } \\
\text { referencia a su autoeficacia se compone por dichas variables. }\end{array}$ \\
\hline 2017 & Cuba & Tejuca, Gutiérrez, Hernández y García & $\begin{array}{l}\text { Estudiar en qué medida el actual proceso de ingreso a la ES contribuye } \\
\text { a la selección de estudiantes de piel blanca, hijos de universitarios, } \\
\text { profesionistas o dirigentes. }\end{array}$ & $\begin{array}{l}\text { Cuantitativa, encuesta a } 94 \% \text { de los estudiantes del último grado de } \\
\text { enseñanza preuniversitaria. }\end{array}$ & $\begin{array}{l}72,5 \% \text { de los estudiantes admitidos y } 69,8 \% \text { de los que aplicaron } \\
\text { a ES son blancos. Si bien el proceso de ingreso a la ES contribuye al } \\
\text { incremento de la proporción de estudiantes con estas características, } \\
\text { la selección se inicia al concluir la enseñanza secundaria. Lo anterior } \\
\text { debido a que los antecedentes académicos tienen un peso determinante } \\
\text { en el ingreso a ES al proporcionar el índice académico que se promedia } \\
\text { con el puntaje de admisión. }\end{array}$ \\
\hline 2017 & México & Reyes & Caracterizar a los estudiantes de dos generaciones de una EN. & $\begin{array}{l}\text { Cuantitativa, encuesta a } 1096 \text { estudiantes para recuperar variables } \\
\text { sociodemográficas, económicas, académicas, profesionales y culturales. }\end{array}$ & $\begin{array}{l}\text { La mayoría son mujeres, ívenenes y solteras }(93 \%) \text {. La escolaridad de } \\
\text { los padres es de nueve años y poco más de } 50 \% \text { de los participantes } \\
\text { reconocen que fue relevante su influencia en la elección de la carrera } \\
\text { docente. }\end{array}$ \\
\hline 2017 & México & Ochoa, Vázquez y González & Identificar rasgos y características de estudiantes universitarios. & $\begin{array}{l}\text { Cuantitativa, encuesta a } 113 \text { estudiantes de nuevo ingreso de la } \\
\text { generación 2014-2018 de la licenciatura en Ciencias de la Educación. }\end{array}$ & $\begin{array}{l}\text { La mayoría de los participantes son mujeres }(81 \%) \text { solteras, cuyos } \\
\text { padres en promedio cuentan con una escolaridad mínima de secundaria } \\
\text { y las sostienen económicamente. }\end{array}$ \\
\hline 2015 & Alemania & Roloff, Klusmann, Lüdtke y Trautwein & $\begin{array}{l}\text { Describi las características personales, cognitivas y demográiticas de } \\
\text { los estudiantes universitarios para compararlas con el gusto académico } \\
\text { hacia las ciencias o las humanidades. }\end{array}$ & $\begin{array}{l}\text { Cuantitativa, de tipo longitudinal, encuesta a } 1463 \text { estudiantes en } \\
\text { dos momentos: al inicio de su último año de bachillerato y durante el } \\
\text { proceso de admisión a la universidad. }\end{array}$ & 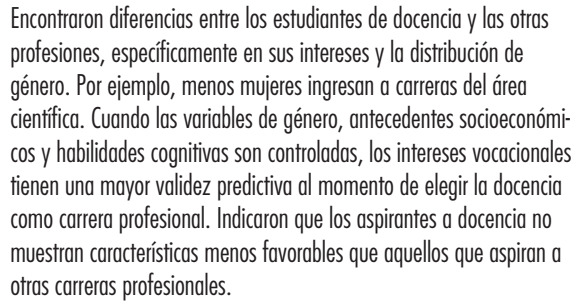 \\
\hline
\end{tabular}


Características de los aspirantes a la Escuela Normal en México. ¿̇En qué condiciones inicia su formación el profesorado de primaria?

\begin{tabular}{|c|c|c|c|c|c|}
\hline AÑo & PAÍS & AUTOR (ES) & OBJETIVO DE LA INVESTIGACIÓN & METODOLOGÍA & RESULTADOS PRINCIPALES \\
\hline 2015 & Reino Unido & Smith y White & $\begin{array}{l}\text { Examinar las características de los estudiantes de diferentes programas } \\
\text { de ES para identificar el rol de la licenciatura estudiada en la posibilidad } \\
\text { de graduarse exitosamente. }\end{array}$ & $\begin{array}{l}\text { Cuantitativa, encuesta a más de } 38000 \text { estudiantes universitarios de } \\
\text { nueve generaciones (2001-2009). }\end{array}$ & $\begin{array}{l}\text { Generaron modelos multivariados al combinar características demográ- } \\
\text { ficas, geográficas y académicas. Destacó la estrecha relación entre los } \\
\text { antecedentes académicos y la posibilidad de graduarse exitosamente. }\end{array}$ \\
\hline 2014 & México & Yañez, Vera y Mungarro & $\begin{array}{l}\text { Evaluar el poder predictivo de las características socioeconómicas y } \\
\text { académicas, sobre el rendimiento académico de los alumnos al conduir } \\
\text { su primer año en la EN. }\end{array}$ & $\begin{array}{l}\text { Cuantitativa, de tipo transversal, al analizar un índice de variables } \\
\text { socioeconómicas, el promedio de preparatoria y el puntaje obtenido } \\
\text { en el Examen Nacional de Ingreso II (EXAN-II) de } 1.903 \text { estudiantes } \\
\text { normalistas. }\end{array}$ & $\begin{array}{l}\text { Encontraron que los estudiantes con mejor rendimiento académico } \\
\text { tienen padres con una mayor escolaridad y mayores posesiones mate- } \\
\text { riales, resultado coincidente con los estudios realizados en contextos } \\
\text { universitarios. Ubicaron al examen de ingreso como el requisito de } \\
\text { admisión que hace la diferencia más amplia entre los aspirantes; el } \\
\text { promedio de preparatoria es la variable con mayor peso predictivo. }\end{array}$ \\
\hline
\end{tabular}


En cuanto al objetivo de los estudios previos se encontró que buscaron describir una serie de características de los participantes y, en algunos casos, superaron la descripción para presentar la asociación entre las características y otras variables como el desempeño académico, la posibilidad de ingreso a la ES y la elección de carrera universitaria. Todos los estudios revisados abordaron el objeto de estudio desde un método cuantitativo, de forma específica a través del uso de cuestionarios estandarizados. Sin embargo el tamaño de la muestra fue variado, desde el uso de muestras representativas hasta la aplicación censal de las técnicas de recolección de datos; por ejemplo generaciones completas de estudiantes o grupos de aspirantes.

En la sección de los resultados, todos los estudios previos revisados coinciden en afirmar que las características de los estudiantes tienen influencia en otros aspectos, por lo que resaltan la importancia que tiene la elaboración de estudios que permitan conocer a los estudiantes universitarios. La información que se recupera con este tipo de investigaciones es necesaria para la construcción de perfiles específicos de los estudiantes y el consecuente diseño de políticas educativas que permitan mejorar el acompañamiento institucional durante sus estudios superiores.

Es preciso clarificar que no se tenía la intención de elaborar un estado de la cuestión, sino presentar una síntesis de la revisión de la literatura para puntualizar elementos comunes en los estudios realizados recientemente y así justificar la relevancia de la investigación.

Esta revisión permitió hacer evidente un vacío en lo que respecta a investigaciones enfocadas en los aspirantes a EN. En este artículo se presentan resultados parciales de un proyecto más amplio orientado a identificar los factores motivaciones presentes en la decisión de estudiar en la EN. El estudio se realizó en Baja California, entidad ubicada al noroeste del país, en la frontera de México con Estados Unidos de América.

En específico este artículo tiene los siguientes objetivos: (1) describir las características laborales, socioeconómicas, familiares y académicas de los aspirantes; (2) identificar diferencias estadísticamente significativas entre las EN participantes, respecto a las características descritas; y (3) identificar diferencias estadísticamente significativas en las características de los aspirantes de acuerdo a la zona donde su ubica la EN (urbana/rural), respecto a las características descritas.

\section{Método}

Se trata de un estudio de tipo descriptivo y transversal, realizado desde el enfoque cuantitativo con la aplicación de una encuesta. El instrumento utilizado fue el Cuestionario de los Factores Motivacionales que influyen en la Elección Profesional de la Carrera Docente (CFAMDOC), que fue diseñado, desarrollado y validado en el marco del proyecto de investigación más amplio que se encuentra en proceso. El análisis de fiabilidad del instrumento resultó favorable $(\alpha=0,948)$.

El cuestionario se compone de dos apartados que integran 122 reactivos. En el primero se incluyen 70 reactivos distribuidos en ocho secciones que se enfocan a recuperar las características contextuales; en el segundo apartado se presentan 52 reactivos para evaluar nueve factores motivacionales que influyen en la elección de la LEPRIM como carrera profesional. En este artículo se analizan algunos de los datos recuperados en el primer apartado del CFAMDOC (Ver Tabla II), en función de los objetivos de investigación planteados. 


\section{Tabla II. Distribución de los reactivos de la primera parte del CFAMDOC}

\begin{tabular}{|c|c|c|}
\hline SECCIÓN & CANTIDAD DE REACTIVOS & VARIABLES \\
\hline \multirow{3}{*}{ Datos generales } & \multirow{3}{*}{5} & Género \\
\hline & & Estado civil \\
\hline & & Edad \\
\hline \multirow{11}{*}{ Características socioeconómicas } & \multirow{11}{*}{13} & Trabajo actual \\
\hline & & Propiedad de la vivienda \\
\hline & & Cantidad de personas que habitan la vivienda \\
\hline & & Cantidad de focos \\
\hline & & Cantidad de habitaciones para dormir \\
\hline & & Espacio apropiado para estudiar \\
\hline & & Servicio de internet \\
\hline & & Dispositivos electrónicos \\
\hline & & Automóvil propio \\
\hline & & Cantidad de libros en casa \\
\hline & & Tipo de servicio médico \\
\hline \multirow{3}{*}{ Características familiares } & \multirow{3}{*}{6} & Máximo grado de estudios de los padres \\
\hline & & Ocupación de los padres \\
\hline & & Existencia familiares profesores \\
\hline \multirow{6}{*}{ Características académicas } & \multirow{6}{*}{12} & Ubicación \\
\hline & & Tipo y modalidad de bachillerato \\
\hline & & Asignaturas reprobadas \\
\hline & & Promedio de bachillerato \\
\hline & & Capacidades en inglés: comprensión lectora, expresión oral, expresión escrita y comprensión auditiva \\
\hline & & Beca en bachillerato \\
\hline
\end{tabular}

Fuente: elaboración propia a partir del CFAMDOC.

La recolección de información para este estudio se realizó en las seis EN que ofrecen la LEPRIM en Baja California, las cuales se identifican con números del uno al seis. El instrumento fue aplicado a 345 aspirantes durante el proceso de selección para ingresar a la LEPRIM (generación 2018-2022); esta cantidad representa $98 \%$ de la totalidad de aspirantes registrados (Ver Tabla III), por lo que el estudio se considera de carácter censal.

Tabla III. Cantidad de participantes por EN

\begin{tabular}{|c|c|c|c|c|}
\hline EN & ZONA & CANTIDAD DE ASPIRANTES REGISTRADOS & $\begin{array}{l}\text { CANTIDAD DE PARTICIPANTES EN EL } \\
\text { ESTUDIO }\end{array}$ & PORCENTAJE DE PARTICIPACIÓN \\
\hline EN1 & Urbana & 60 & 57 & $95 \%$ \\
\hline EN2 & Rural & 49 & 49 & $100 \%$ \\
\hline EN3 & Rural & 51 & 51 & $100 \%$ \\
\hline EN4 & Urbana & 103 & 99 & $96 \%$ \\
\hline EN5 & Urbana & 40 & 39 & $98 \%$ \\
\hline \multirow[t]{2}{*}{ EN6 } & Rural & 50 & 50 & $100 \%$ \\
\hline & Total & 353 & 345 & $98 \%$ \\
\hline
\end{tabular}

Fuente: elaboración propia. 
Como puede verse en la Tabla III, la mitad de las instituciones se ubica en comunidades rurales y el resto en zonas urbanas del estado. En la sección de resultados de este artículo se distinguen las variables analizadas que mostraron diferencias estadísticamente significativas al agrupar a los participantes de acuerdo con esta condición.

El acceso a los participantes para la recolección de información inició con la negociación con la autoridad educativa estatal representada por la Dirección de Educación Superior para Profesionales de la Educación (DESPE), quien autorizó la ejecución del proyecto y la participación de los aspirantes. El periodo de aplicación del instrumento de recolección de datos fue del 5 de marzo al 4 de mayo de 2018, a través de la plataforma digital Lime Survey. En el cuestionario se incluyó la explicación del proyecto y se especificó el resguardo confidencial de la información, además de asegurar que las respuestas otorgadas no tendrían ningún impacto en el ingreso a la EN. Nunca se planteó como obligatoria la participación de los aspirantes, sin embargo, ninguno se rehusó a responder el cuestionario.

Una vez que se recolectaron las respuestas de los participantes, se generó una base de datos en el Programa SPSS (versión 21) para realizar diferentes análisis estadísticos. En primer término se revisaron las codificaciones de todas las respuestas y se determinaron aquellas a las que correspondía un valor perdido, con lo que se finalizó el acomodo de la base de datos para dar inicio al análisis correspondiente. Se realizaron distintas pruebas de estadística descriptiva, como cálculo de media y desviación estándar; e inferencial, como Prueba t y ANOVA.

\section{Resultados}

A partir del análisis de los datos recolectados se presentan a continuación los resultados correspondientes a las características de los aspirantes a la LEPRIM en Baja California, así como la precisión de aquellas diferencias estadísticamente significativas que pudieron identificarse al clasificar a los participantes en seis grupos de acuerdo a la EN a la que aspiraban ingresar y en dos grupos de acuerdo a la zona donde se ubica la escuela: urbana y rural.

\subsection{Características de los aspirantes}

De los 345 aspirantes que participaron en el estudio 79\% son mujeres, lo que corresponde a la tendencia internacional (Gratacós, 2014) y mexicana (Reyes, 2017) que caracteriza a la docencia como una profesión femenina. En promedio tienen 19 años de edad (DE: 4,20), donde la edad mínima registrada fue 16 años y la máxima 43 años de edad. Además, se encontró que $90 \%$ afirman ser solteros, del resto, $5 \%$ está casado y $5 \%$ en una relación de unión libre. En la Tabla IV se presenta una síntesis de los datos generales de los participantes.

\section{Tabla IV. Datos generales de los aspirantes}

\begin{tabular}{lccccccc}
\hline & $\begin{array}{c}\text { GENERAL } \\
(\mathbf{N}=345)\end{array}$ & $\begin{array}{c}\text { EN1 } \\
(\mathbf{N}=57)\end{array}$ & $\begin{array}{c}\text { EN2 } \\
(\mathbf{N}=49)\end{array}$ & $\begin{array}{c}\text { EN3 } \\
(\mathbf{N}=51)\end{array}$ & $\begin{array}{c}\text { EN4 } \\
(\mathbf{N}=99)\end{array}$ & $\begin{array}{c}\text { EN5 } \\
(\mathbf{N}=39)\end{array}$ & $\begin{array}{c}\text { EN6 } \\
(\mathbf{N}=50)\end{array}$ \\
\hline Género & & & & & & & \\
\hline Femenino & $79 \%$ & $77 \%$ & $78 \%$ & $69 \%$ & $86 \%$ & $79 \%$ & $80 \%$ \\
\hline Masculino & $21 \%$ & $23 \%$ & $22 \%$ & $29 \%$ & $14 \%$ & $21 \%$ & $20 \%$ \\
\hline Otros & $0.3 \%$ & $0 \%$ & $0 \%$ & $2 \%$ & $0 \%$ & $0 \%$ & $0 \%$ \\
\hline Estado civil & & & & & & & \\
\hline Soltero & $88 \%$ & $93 \%$ & $98 \%$ & $90 \%$ & $75 \%$ & $85 \%$ & $96 \%$ \\
\hline Unión libre & $5 \%$ & $0 \%$ & $2 \%$ & $4 \%$ & $9 \%$ & $13 \%$ & $0 \%$ \\
\hline Casado & $5 \%$ & $7 \%$ & $0 \%$ & $6 \%$ & $8 \%$ & $3 \%$ & $4 \%$ \\
\hline
\end{tabular}




\begin{tabular}{llllllll}
\hline & $\begin{array}{c}\text { GENERAL } \\
(\mathbf{N}=345)\end{array}$ & $\begin{array}{c}\text { EN1 } \\
(\mathbf{N}=57)\end{array}$ & $\begin{array}{c}\text { EN2 } \\
(\mathbf{N}=49)\end{array}$ & $\begin{array}{c}\text { EN3 } \\
(\mathbf{N}=51)\end{array}$ & $\begin{array}{c}\text { EN4 } \\
(\mathbf{N}=99)\end{array}$ & $\begin{array}{c}\text { EN5 } \\
(\mathbf{N}=39)\end{array}$ & $\begin{array}{c}\text { EN6 } \\
(\mathbf{N}=50)\end{array}$ \\
\hline Categoría de edad & & & & & & & \\
\hline $16-19$ años & $71 \%$ & $77 \%$ & $82 \%$ & $76 \%$ & $63 \%$ & $67 \%$ & $68 \%$ \\
\hline $20-23$ años & $16 \%$ & $12 \%$ & $12 \%$ & $8 \%$ & $21 \%$ & $21 \%$ & $18 \%$ \\
\hline $24-27$ años & $6 \%$ & $7 \%$ & $2 \%$ & $2 \%$ & $6 \%$ & $10 \%$ & $10 \%$ \\
\hline $28-31$ años & $4 \%$ & $2 \%$ & $2 \%$ & $10 \%$ & $6 \%$ & $3 \%$ & $0 \%$ \\
\hline $32-35$ años & $2 \%$ & $2 \%$ & $0 \%$ & $2 \%$ & $3 \%$ & $0 \%$ & $2 \%$ \\
\hline Otros 36-43 años & $3 \%$ & $2 \%$ & $2 \%$ & $4 \%$ & $4 \%$ & $0 \%$ & $4 \%$ \\
\hline Edad promedio & 19,8 & $\mathbf{1 9 , 3}$ & $\mathbf{1 9 , 2}$ & $\mathbf{2 0 , 2}$ & $\mathbf{2 0 , 2}$ & $\mathbf{1 9 , 6}$ & $\mathbf{1 9 , 9}$ \\
\hline
\end{tabular}

Fuente: elaboración propia.

Un dato que se considera relevante destacar en esta sección corresponde a la edad de los aspirantes. Aunque la mayoría en los seis casos estudiados se ubica en la categoría considerada edad regular para ingresar a la ES, entre los 17 y 19 años, se presentan aspirantes de otras edades. Por ejemplo, en la EN2 se encontró que $10 \%$ de los aspirantes tienen entre 28 y 30 años de edad. La diversidad en edad debe ser considerada por las EN como un elemento característico de los aspirantes, al presentar necesidades distintas que los alumnos en edad regular.

\subsubsection{Características laborales}

En la Tabla $\mathrm{V}$ se muestran los resultados generales por EN de los reactivos correspondientes a las características laborales, destaca que $62 \%$ expresó no contar con un empleo. De la población que trabaja, se encontró que $51 \%$ de los aspirantes de la EN4 lo hace más de 20 horas a la semana, al igual que $43 \%$ de la EN6 y $38 \%$ de la EN2. Por otra parte, $40 \%$ de los aspirantes de la EN3 y $47 \%$ de la EN5 trabaja menos de 10 horas semanales.

Tabla V. Características laborales de los aspirantes

\begin{tabular}{|c|c|c|c|c|c|c|c|}
\hline & $\begin{array}{l}\text { GENERAL } \\
(\mathrm{N}=345)\end{array}$ & $\begin{array}{c}\text { EN1 } \\
(\mathrm{N}=57)\end{array}$ & $\begin{array}{c}\text { EN2 } \\
(\mathrm{N}=49)\end{array}$ & $\begin{array}{c}\text { EN3 } \\
(\mathrm{N}=51)\end{array}$ & $\begin{array}{c}\text { EN4 } \\
(\mathrm{N}=99)\end{array}$ & $\begin{array}{c}\text { EN5 } \\
(\mathrm{N}=39)\end{array}$ & $\begin{array}{c}\text { EN6 } \\
(\mathrm{N}=50)\end{array}$ \\
\hline \multicolumn{8}{|l|}{ Trabajo actual } \\
\hline No & $62 \%$ & $67 \%$ & $67 \%$ & $57 \%$ & $65 \%$ & $56 \%$ & $58 \%$ \\
\hline Sí & $37 \%$ & $33 \%$ & $33 \%$ & $43 \%$ & $35 \%$ & $44 \%$ & $42 \%$ \\
\hline Menos de 10 horas & $27 \%$ & $26 \%$ & $6 \%$ & $40 \%$ & $20 \%$ & $47 \%$ & $24 \%$ \\
\hline Entre 10 y 20 horas & $21 \%$ & $37 \%$ & $31 \%$ & $35 \%$ & $14 \%$ & $0 \%$ & $14 \%$ \\
\hline Más de 20 horas & $36 \%$ & $26 \%$ & $38 \%$ & $10 \%$ & $51 \%$ & $35 \%$ & $43 \%$ \\
\hline Solo fines de semana & $16 \%$ & $11 \%$ & $25 \%$ & $15 \%$ & $14 \%$ & $18 \%$ & $19 \%$ \\
\hline Percepción de salario & $77 \%$ & $74 \%$ & $75 \%$ & $70 \%$ & $86 \%$ & $76 \%$ & $71 \%$ \\
\hline Relacionado a la docencia & $11 \%$ & $5 \%$ & $13 \%$ & $10 \%$ & $23 \%$ & $6 \%$ & $0 \%$ \\
\hline
\end{tabular}

Fuente: elaboración propia.

Mudavanhu (2015) destaca la relevancia de la experiencia laboral en la elección de la docencia como carrera profesional. En este sentido, solo $11 \%$ de los aspirantes que trabajan mencionaron que la actividad económica en la que se desempeñan está relacionada de alguna manera a la docencia, por lo que se infiere que la experiencia laboral no apoyó necesariamente la decisión vocacional.

\subsubsection{Características socioeconómicas}

Las características socioeconómicas generales de los participantes se presentan en la Tabla VI. 
En cuanto a la propiedad de la vivienda familiar de los aspirantes, $85 \%$ de los encuestados respondieron que su casa es propia, en la cual viven en promedio cuatro personas. La cantidad de personas que comparten la vivienda fue dividida entre el número de habitaciones para generar el nivel de hacinamiento (Spicker et al., 2009), que en general resultó de 1,83 personas por habitación. En el mismo rubro de la vivienda se recuperó la cantidad de focos, contar con un espacio destinado para el estudio y contar con el servicio de internet.

En cuanto a la posesión de dispositivos electrónicos, la mayoría de los participantes cuenta con celular inteligente (88\%), mientras que 54\% tiene una computadora portátil. Este dato cobra gran importancia para las EN, pues en la actualidad los recursos tecnológicos son indispensables para el adecuado desarrollo de las actividades académicas propuestas en el curso del programa educativo de la LEPRIM.

Tabla VI. Características socioeconómicas de los aspirantes

\begin{tabular}{|c|c|c|c|c|c|c|c|}
\hline & $\begin{array}{l}\text { GENERAL } \\
(\mathrm{N}=345)\end{array}$ & $\begin{array}{c}\text { EN1 } \\
(\mathrm{N}=57)\end{array}$ & $\begin{array}{c}\text { EN2 } \\
(\mathrm{N}=49)\end{array}$ & $\begin{array}{c}\text { EN3 } \\
(\mathrm{N}=51)\end{array}$ & $\begin{array}{c}\text { EN4 } \\
(\mathrm{N}=99)\end{array}$ & $\begin{array}{c}\text { EN5 } \\
(\mathrm{N}=39)\end{array}$ & $\begin{array}{c}\text { EN6 } \\
(\mathrm{N}=50)\end{array}$ \\
\hline \multicolumn{8}{|l|}{ Vivienda familiar } \\
\hline Propia & $85 \%$ & $89 \%$ & $90 \%$ & $86 \%$ & $82 \%$ & $72 \%$ & $92 \%$ \\
\hline Rentada & $10 \%$ & $5 \%$ & $4 \%$ & $6 \%$ & $15 \%$ & $21 \%$ & $4 \%$ \\
\hline Prestada & $5 \%$ & $5 \%$ & $6 \%$ & $8 \%$ & $3 \%$ & $8 \%$ & $4 \%$ \\
\hline Personas en el hogar & 4,5 & 4,3 & 4,3 & 4,4 & 4,4 & 4,4 & 5,2 \\
\hline Recámaras & 2,6 & 2,5 & 2,6 & 2,7 & 2,7 & 2,5 & 2,6 \\
\hline Hacinamiento & 1,83 & 1,78 & 1,70 & 1,82 & 1,77 & 1,82 & 2,14 \\
\hline Focos & 8,1 & 9,1 & 7,6 & 7,5 & 8,4 & 8,4 & 7,6 \\
\hline Espacio para estudiar & $66 \%$ & $58 \%$ & $67 \%$ & $67 \%$ & $72 \%$ & $67 \%$ & $60 \%$ \\
\hline Internet en casa & $80 \%$ & $84 \%$ & $78 \%$ & $63 \%$ & $88 \%$ & $90 \%$ & $72 \%$ \\
\hline \multicolumn{8}{|l|}{ Dispositivos electrónicos } \\
\hline Computadora portátil & $54 \%$ & $65 \%$ & $49 \%$ & $47 \%$ & $60 \%$ & $46 \%$ & $50 \%$ \\
\hline Celular inteligente & $88 \%$ & $95 \%$ & $82 \%$ & $86 \%$ & $93 \%$ & $90 \%$ & $76 \%$ \\
\hline Computadora personal & $27 \%$ & $26 \%$ & $39 \%$ & $24 \%$ & $23 \%$ & $31 \%$ & $22 \%$ \\
\hline Tableta & $14 \%$ & $19 \%$ & $2 \%$ & $10 \%$ & $16 \%$ & $15 \%$ & $16 \%$ \\
\hline Ninguno & $3 \%$ & $2 \%$ & $4 \%$ & $6 \%$ & $1 \%$ & $0 \%$ & $10 \%$ \\
\hline Otro & $2 \%$ & $0 \%$ & $2 \%$ & $0 \%$ & $2 \%$ & $3 \%$ & $4 \%$ \\
\hline Automóvil propio & $19 \%$ & $21 \%$ & $35 \%$ & $25 \%$ & $16 \%$ & $8 \%$ & $12 \%$ \\
\hline Libros en casa & 28 & 21 & 35 & 25 & 16 & 8 & 12 \\
\hline \multicolumn{8}{|l|}{ Servicio médico } \\
\hline Privado & $10 \%$ & $9 \%$ & $12 \%$ & $8 \%$ & $9 \%$ & $15 \%$ & $8 \%$ \\
\hline Público & $90 \%$ & $89 \%$ & $88 \%$ & $92 \%$ & $91 \%$ & $85 \%$ & $92 \%$ \\
\hline
\end{tabular}

Fuente: elaboración propia.

También se recuperó que únicamente 19\% de los aspirantes cuentan con un automóvil propio, aspecto en el que destaca la EN2, pues $35 \%$ de sus aspirantes poseen este medio de transporte. En cuanto a la posesión de libros en casa el promedio general es de 28 , aunque los aspirantes de la EN5 mencionaron tener en promedio solamente ocho ejemplares bibliográficos. En relación al tipo de servicio médico utilizado por las familias, $90 \%$ de los aspirantes acuden al servicio público. Estos datos coinciden con la descripción realizada por Reyes (2017) de estudiantes normalistas mexicanos. 
Identificar este tipo de información sobre los antecedentes socioeconómicos de los participantes es sumamente relevante, debido a la influencia que pueden tener «las desigualdades socioeconómicas y culturales (...) en la entrada o en el rendimiento académico» (Herrera, 2019: 10).

\subsubsection{Características familiares}

Debido a que «las familias suponen un soporte central para la educación escolar de sus hijos》(Alonso, 2014: 395), se consideró relevante recuperar información respecto al contexto familiar de los participantes, por ejemplo, el grado máximo de estudios de sus padres. Al respecto, se encontró que el porcentaje mayor de los padres de familia (30\%) estudió hasta la secundaria, situación similar en la mayoría de las EN participantes. Destaca que el grado máximo de estudios de $42 \%$ de los padres de los aspirantes a ingresar a la EN6 es la primaria; mientras que $25 \%$ de los padres de los aspirantes de la EN1 cuenta con licenciatura y otro 25\% tiene educación secundaria.

En general, solo 12\% de los participantes expresó que sus padres estudiaron la universidad. Esta cifra está por debajo de los resultados reportados en España donde «algo más del $60 \%$ de estudiantes aspirantes a obtener un titulo universitario, superarian, en el caso de llegar a lograrlo, el nivel de estudios de sus progenitores》 (Herrera, 2019: 20). Este dato brinda evidencia de la condición de desventaja en la que se encuentra esta parte de la población mexicana frente a la sociedad española.

Al igual que en el caso de los padres, el máximo grado de estudio de las madres de familia es la secundaria (33\%). Esta situación fue coincidente en todas las EN, a excepción de la EN6, donde el porcentaje mayor de madres (44\%) estudió únicamente la educación primaria. Cabe recordar que esta situación se repite en el caso de los padres de familia de la misma institución. A pesar de considerarse un nivel de estudios bajo, la población descrita supera la escolaridad reportada por Reyes (2017), quienes contaban con nueve años de escolaridad de los padres de estudiantes normalistas de la Ciudad de México.

Otro elemento que se consideró importante en la caracterización de los aspirantes al profesorado es la presencia de familiares en el magisterio, pues se valora como una influencia relevante en la elección profesional. Los participantes del estudio reportaron que $65 \%$ de ellos efectivamente tienen profesores en sus familias. La EN3 destaca en este rubro, pues $76 \%$ de sus aspirantes expresaron tener familiares profesores. Es posible inferir que los familiares profesores tuvieron cierto grado de influencia en la decisión de los aspirantes, al recomendarles la profesión docente, dato coincidente con el recuperado por Zamora y Cabrera (2015).

Dado que se recuperó que el promedio de escolaridad de los padres es la educación secundaria, se consideró necesario identificar el parentesco de los familiares maestros reportados por los aspirantes. En su mayoría (59\%) respondieron que son tíos o primos quienes tienen esta profesión en sus familias (Ver Tabla VII).

Tabla VII. Parentesco de los familiares en el magisterio de los aspirantes

\begin{tabular}{|c|c|c|c|c|c|c|c|}
\hline & $\begin{array}{l}\text { GENERAL } \\
(\mathrm{N}=345)\end{array}$ & $\begin{array}{c}\text { EN1 } \\
(\mathrm{N}=57)\end{array}$ & $\begin{array}{c}\text { EN2 } \\
(\mathrm{N}=49)\end{array}$ & $\begin{array}{c}\text { EN3 } \\
(\mathrm{N}=51)\end{array}$ & $\begin{array}{c}\text { EN4 } \\
(\mathrm{N}=99)\end{array}$ & $\begin{array}{c}\text { EN5 } \\
(\mathrm{N}=39)\end{array}$ & $\begin{array}{c}\text { EN6 } \\
(\mathrm{N}=50)\end{array}$ \\
\hline Familiares profesores & $65 \%$ & $63 \%$ & $65 \%$ & $76 \%$ & $62 \%$ & $64 \%$ & $62 \%$ \\
\hline Papá/mamá & $16 \%$ & $31 \%$ & $16 \%$ & $13 \%$ & $13 \%$ & $20 \%$ & $7 \%$ \\
\hline Hermanos (as) & $17 \%$ & $14 \%$ & $25 \%$ & $18 \%$ & $10 \%$ & $12 \%$ & $26 \%$ \\
\hline Tíos/primos (as) & $59 \%$ & $47 \%$ & $56 \%$ & $69 \%$ & $64 \%$ & $60 \%$ & $55 \%$ \\
\hline
\end{tabular}




\begin{tabular}{lccccccc}
\hline & $\begin{array}{c}\text { GENERAL } \\
(\mathbf{N}=345)\end{array}$ & $\begin{array}{c}\text { EN1 } \\
(\mathbf{N}=57)\end{array}$ & $\begin{array}{c}\text { EN2 } \\
(\mathbf{N}=49)\end{array}$ & $\begin{array}{c}\text { EN3 } \\
(\mathbf{N}=51)\end{array}$ & $\begin{array}{c}\text { EN4 } \\
(\mathbf{N}=99)\end{array}$ & $\begin{array}{c}\text { EN5 } \\
(\mathbf{N}=39)\end{array}$ & $\begin{array}{c}\text { EN6 } \\
(\mathbf{N}=50)\end{array}$ \\
\hline Abuelos (as) & $5 \%$ & $6 \%$ & $3 \%$ & $0 \%$ & $3 \%$ & $8 \%$ & $10 \%$ \\
\hline Esposo (a) & $2 \%$ & $3 \%$ & $0 \%$ & $0 \%$ & $5 \%$ & $0 \%$ & $3 \%$ \\
\hline Otro & $1 \%$ & $0 \%$ & $0 \%$ & $0 \%$ & $5 \%$ & $0 \%$ & $0 \%$ \\
\hline
\end{tabular}

Fuente: elaboración propia.

\subsubsection{Indice Socioeconómico (ISEC)}

Se calculó el Índice Socioeconómico (ISEC) a través del procedimiento de Análisis de Componentes Principales; se unieron los resultados de 13 de los reactivos del CFAMDOC (cantidad de focos, espacio de estudio, servicio de internet, dispositivos electrónicos, automóvil propio, cantidad de libros en casa, tipo de servicio médico, grado de estudios y ocupación de los padres) al nivel de hacinamiento.

Calcular el ISEC permitió comparar la posición socioeconómica de los participantes bajo un mismo criterio, ante lo que resultaron puntajes dentro del rango: -2,14 a 3,44. Los puntajes en la parte negativa de la escala representan condiciones socioeconómicas desfavorables. En el Gráfico 1 se muestra la media del ISEC por EN.

En general, el ISEC de los aspirantes es desfavorable. Este dato debe ser considerado tanto por las autoridades institucionales de las EN participantes como por las autoridades estatales para el diseño de medidas compensatorias que apoyen el tránsito de los estudiantes por los estudios normalistas, y así evitar que el factor económico los lleve a la deserción temporal o definitiva.

Además, es necesario considerar que de acuerdo con Yañez y colaboradores (2014) los aspirantes admitidos a la EN tienen padres con mayor grado escolar y más posesiones materiales, por lo que las características socioeconómicas descritas colocan a los participantes señalados en condiciones de desventaja.

\section{Gráfico 1. Promedio del ISEC de los aspirantes}

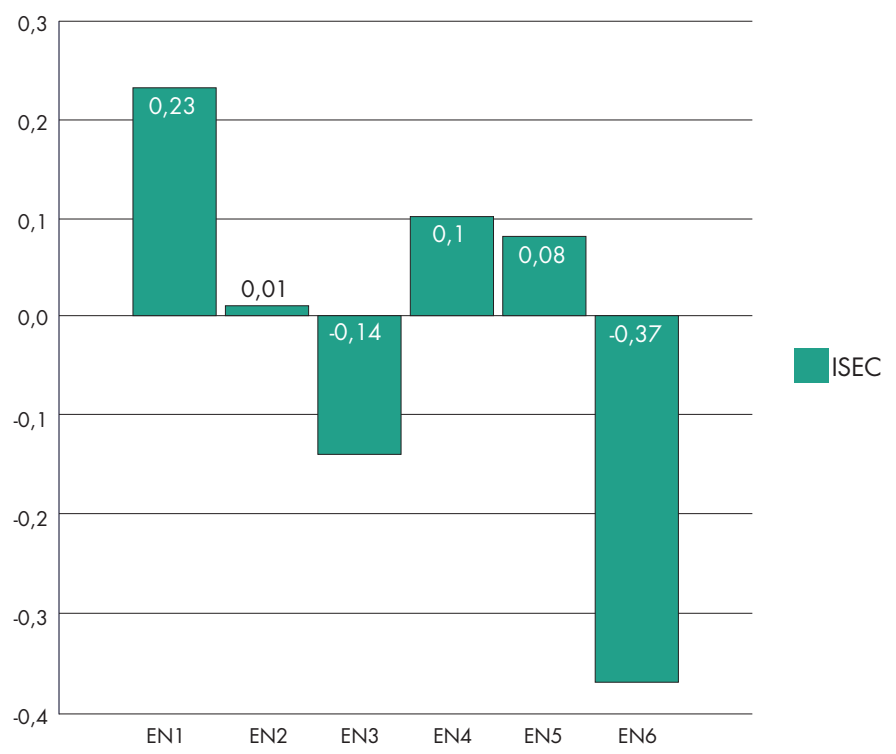

Fuente: elaboración propia a partir de PISA 2015. 


\subsubsection{Características académicas}

En la Tabla VIII se presentan los porcentajes generales de los reactivos correspondientes a las características académicas de los aspirantes a ingresar a la LEPRIM. En cuanto a la ubicación del bachillerato de egreso de los participantes, se encontró que en la mayoría de los casos, $68 \%$ general, se encuentra en la misma localidad donde se ubica la EN en donde realizan el proceso de selección. A excepción de los aspirantes a ingresar a la EN3, pues $51 \%$ de ellos estudiaron el bachillerato en otra localidad. El contar con esta información permitirá a las autoridades institucionales identificar algunas necesidades particulares de los estudiantes foráneos.

También se recuperó el tipo de bachillerato del que egresaron los participantes. En México, dicho nivel educativo se ofrece bajo tres modelos distintos: bachillerato general, que prepara a los estudiantes para ingresar a la ES por su carácter propedéutico; bachillerato tecnológico y profesional técnico, ambos modelos preparan a los estudiantes tanto para ingresar a la ES como para insertarse en el mercado laboral. En el caso de este estudio se encontró una escasa participación de aspirantes provenientes de bachillerato profesional técnico. El porcentaje mayor en tres de las EN se ubica en el bachillerato general, a excepción de la EN2, EN5 y EN6, donde la mayoría de aspirantes egresaron de bachilleratos del tipo tecnológico. Esta información se considera relevante, pues permitirá a las instituciones formadoras de docentes enfocar sus esfuerzos de difusión en las escuelas preparatorias de tipo general y tecnológico. En el mismo sentido, la modalidad de bachillerato en la que estudiaron los aspirantes es escolarizada casi en su totalidad.

Los participantes reportaron un nivel muy bajo de asignaturas reprobadas en sus estudios de educación media superior. Además, el promedio de egreso de los aspirantes es favorable, 8,7 en general. Cabe señalar que es requisito de ingreso a la EN un promedio de calificación mínimo de 8 sobre 10 en el bachillerato. Este resultado coincide con lo expuesto por Yañez et al., (2014), quienes afirman que las calificaciones de bachillerato con las que ingresan los estudiantes a la EN son cercanas a la excelencia.

\section{Tabla VIII. Características académicas de los aspirantes}

\begin{tabular}{|c|c|c|c|c|c|c|c|}
\hline & $\begin{array}{l}\text { GENERAL } \\
(\mathrm{N}=345)\end{array}$ & $\begin{array}{c}\text { EN1 } \\
(\mathrm{N}=57)\end{array}$ & $\begin{array}{c}\text { EN2 } \\
(\mathrm{N}=49)\end{array}$ & $\begin{array}{c}\text { EN3 } \\
(\mathrm{N}=51)\end{array}$ & $\begin{array}{c}\text { EN4 } \\
(\mathrm{N}=99)\end{array}$ & $\begin{array}{c}\text { EN5 } \\
(\mathrm{N}=39)\end{array}$ & $\begin{array}{c}\text { EN6 } \\
(\mathrm{N}=50)\end{array}$ \\
\hline \multicolumn{8}{|l|}{ Bachillerato local } \\
\hline Sí & $68 \%$ & $67 \%$ & $55 \%$ & $49 \%$ & $76 \%$ & $77 \%$ & $80 \%$ \\
\hline No & $32 \%$ & $33 \%$ & $45 \%$ & $51 \%$ & $24 \%$ & $23 \%$ & $20 \%$ \\
\hline \multicolumn{8}{|l|}{ Tipo de bachillerato } \\
\hline General & $48 \%$ & $58 \%$ & $43 \%$ & $75 \%$ & $47 \%$ & $28 \%$ & $32 \%$ \\
\hline Tecnológico & $45 \%$ & $33 \%$ & $49 \%$ & $25 \%$ & $43 \%$ & $59 \%$ & $66 \%$ \\
\hline Profesional técnico & $7 \%$ & $9 \%$ & $8 \%$ & $0 \%$ & $9 \%$ & $13 \%$ & $2 \%$ \\
\hline \multicolumn{8}{|l|}{ Modalidad de bachillerato } \\
\hline Escolarizada & $94 \%$ & $96 \%$ & $88 \%$ & $94 \%$ & $96 \%$ & $95 \%$ & $90 \%$ \\
\hline Semiescolarizada & $2 \%$ & $0 \%$ & $2 \%$ & $2 \%$ & $1 \%$ & $3 \%$ & $6 \%$ \\
\hline En línea & $1 \%$ & $2 \%$ & $0 \%$ & $0 \%$ & $1 \%$ & $0 \%$ & $0 \%$ \\
\hline Abierta & $4 \%$ & $2 \%$ & $10 \%$ & $4 \%$ & $2 \%$ & $3 \%$ & $4 \%$ \\
\hline Asignaturas reprobadas en bachillerato & 0,3 & 0.2 & 0,1 & 0,3 & 0,3 & 0,2 & 0,4 \\
\hline Promedio de bachillerato & 8,7 & 8,8 & 8,7 & 8,6 & 8,7 & 8,8 & 8,7 \\
\hline Beca en bachillerato & $37 \%$ & $35 \%$ & $39 \%$ & $33 \%$ & $31 \%$ & $41 \%$ & $46 \%$ \\
\hline Escasos recursos económicos & $56 \%$ & $70 \%$ & $47 \%$ & $47 \%$ & $45 \%$ & $44 \%$ & $83 \%$ \\
\hline
\end{tabular}




\begin{tabular}{lccccccc}
\hline & $\begin{array}{c}\text { GENERAL } \\
(\mathbf{N}=345)\end{array}$ & $\begin{array}{c}\text { EN1 } \\
(\mathbf{N}=57)\end{array}$ & $\begin{array}{c}\text { EN2 } \\
(\mathbf{N}=49)\end{array}$ & $\begin{array}{c}\text { EN3 } \\
(\mathbf{N}=51)\end{array}$ & $\begin{array}{c}\text { EN4 } \\
(\mathbf{N}=99)\end{array}$ & $\begin{array}{c}\text { EN5 } \\
(\mathbf{N}=39)\end{array}$ & $\begin{array}{c}\text { EN6 } \\
(\mathbf{N}=50)\end{array}$ \\
\hline Excelencia académica & $17 \%$ & $30 \%$ & $21 \%$ & $18 \%$ & $16 \%$ & $25 \%$ & $0 \%$ \\
\hline Deportiva & $8 \%$ & $0 \%$ & $0 \%$ & $0 \%$ & $0 \%$ & $6 \%$ & $0 \%$ \\
\hline Indígena & $0 \%$ & $0 \%$ & $0 \%$ & $0 \%$ & $0 \%$ & $0 \%$ & $0 \%$ \\
\hline No recuerdo el tipo & $22 \%$ & $10 \%$ & $16 \%$ & $29 \%$ & $32 \%$ & $31 \%$ & $13 \%$ \\
\hline Otro & $7 \%$ & $10 \%$ & $16 \%$ & $6 \%$ & $7 \%$ & $0 \%$ & $4 \%$ \\
\hline
\end{tabular}

Fuente: elaboración propia.

Asimismo, se les preguntó a los participantes si contaron con beca durante sus estudios de bachillerato, en general $37 \%$ de ellos respondieron afirmativamente. En la EN6 se presentó el porcentaje más alto, pues $46 \%$ de sus aspirantes mencionaron haber contado con algún apoyo económico de esta naturaleza en la educación media superior. En el mismo sentido, los participantes que el tipo de beca con la que contaron, en su mayoría, fue por escasos recursos económicos.

\subsection{Diferencias estadísticamente significativas por EN}

Se analizaron las respuestas de los participantes agrupados por la EN a la que aspiran ingresar a través de la prueba estadística ANOVA. En la Tabla IX se presentan los resultados de las dos variables que presentaron diferencias estadísticamente significativas: años de escolaridad del padre y el puntaje obtenido en el EXANI-II. Además, se ubicaron diferencias en las variables ISEC y años de escolaridad de la madre, sin embargo en estos casos el resultado de la Prueba de Levene no fue significativo, por lo que no es posible asumir la igualdad de varianzas entre los grupos comparados y el resultado del Estadístico F no puede tomarse en cuenta, aunque resultó significativo.

En las cuatro variables identificadas los aspirantes de la EN1 obtuvieron un puntaje mayor. Los participantes de esa institución tienen un ISEC mayor al resto $(\bar{X}=0,23, \mathrm{DE}=1,05)$, sus madres tienen más años de escolaridad ( $\bar{X}=11,42, \mathrm{DE}=4,32)$, al igual que sus padres $(\bar{X}=12,54, \mathrm{DE}=3,81)$ y obtuvieron un puntaje mayor en el EXANI-II ( $\bar{X}=1044,38, \mathrm{DE}=93,59)$. Dicho examen evalúa pensamiento matemático, pensamiento analítico, estructura de la lengua y comprensión lectora, y es administrado por el Centro Nacional de Evaluación para la Educación Superior (CENEVAL). El puntaje establecido de la prueba se presenta en un rango entre 700 y 1300 puntos, con un puntaje medio esperado de 1000 puntos. En Baja California se señaló como límite aprobatorio un puntaje de 950 que determina la admisión de los aspirantes.

Por el contrario, destacan los resultados de la EN6, pues presentan características desfavorables en casi todos los rubros analizados. Por ejemplo, es la única EN que presenta aspirantes en condición de hacinamiento (2,14 personas por habitación), es decir, con una proporción de habitantes mayor a lo establecido internacionalmente para determinar esta situación. Spicker et al., (2009) sostienen que una proporción mayor a dos personas por habitación en la vivienda representa hacinamiento.

De igual manera, esta EN es la que presenta el ISEC menor $(-0,37)$ y donde el promedio de escolaridad de ambos padres de familia es menor a los nueve años, lo que los ubica en la secundaria inconclusa como grado máximo de escolaridad. Estas características contextuales colocan a los aspirantes a la LEPRIM en la EN6 en una condición desfavorable frente a «sus homólogos provenientes de las clases medias y superiores» (Alonso, 2014: 395), lo que se refleja en los puntajes obtenidos en el EXANI-II. Como 
puede verse en la Tabla IX, la EN6 es la que tiene un promedio menor en este rubro $(\bar{X}=985,07$, $\mathrm{DE}=93,26$ ), que si bien no se considera una evaluación tan alejada al límite esperado, sí se muestra por debajo de la EN1, que tiene el puntaje más alto $(\bar{X}=1044,38, \mathrm{DE}=93,59)$. Estos resultados también se presentan en la EN2 ( $\bar{X}=983,17, \mathrm{DE}=80,09)$, que de igual manera muestra condiciones de vulnerabilidad en varios de los aspectos analizados como el ISEC (Yáñez et. al., 2014).

Esta situación debe ser atendida por las autoridades educativas estatales e institucionales a través de la implementación de programas compensatorios que permitan subsanar, en la medida de lo posible, las carencias que presentan los aspirantes de manera que no sean un elemento que pueda llevarlos a la deserción durante sus estudios normalistas.

Tabla IX. Variables contextuales con diferencia significativa entre las EN participantes

\begin{tabular}{|c|c|c|c|c|c|c|c|c|c|c|c|}
\hline \multirow{4}{*}{ SECCIÓN } & \multirow{4}{*}{ VARIABLE } & \multirow{2}{*}{\multicolumn{6}{|c|}{ GRUPOS }} & \multicolumn{4}{|c|}{ RESULTADOS ESTADÍSTICOS } \\
\hline & & & & & & & & $\begin{array}{l}\text { DIFER } \\
\text { DE VA }\end{array}$ & $\begin{array}{l}\text { ENCIA } \\
\text { ?IANZA }\end{array}$ & $\begin{array}{l}\text { DIFERI } \\
\text { DE MI }\end{array}$ & $\begin{array}{l}\text { ENCIA } \\
\text { EDIAS }\end{array}$ \\
\hline & & $\begin{array}{l}\text { EN1 } \\
\text { MEDIA }\end{array}$ & $\begin{array}{c}\text { EN2 } \\
\text { MEDIA }\end{array}$ & $\begin{array}{c}\text { EN3 } \\
\text { MEDIA } \\
\text { (DE) }\end{array}$ & $\begin{array}{l}\text { EN4 } \\
\text { MEDIA } \\
\text { (DE) }\end{array}$ & $\begin{array}{l}\text { EN5 } \\
\text { MEDIA } \\
\text { (DE) }\end{array}$ & $\begin{array}{l}\text { EN6 } \\
\text { MEDIA } \\
\text { (DE) }\end{array}$ & \multirow[t]{2}{*}{$\begin{array}{l}\text { Estadístico } \\
\text { de Levene }\end{array}$} & \multirow[t]{2}{*}{ Significancia } & \multirow[t]{2}{*}{ Estadístico $\mathrm{F}$} & \multirow[t]{2}{*}{ Significancia } \\
\hline & & $(\mathrm{N}=57)$ & $(\mathrm{N}=49)$ & $(\mathrm{N}=51)$ & ( $N=99)$ & $(\mathrm{N}=39)$ & $(\mathrm{N}=50)$ & & & & \\
\hline \multirow{2}{*}{ Socio-económicas } & \multirow{2}{*}{ ISEC } & 0,23 & $-0,01$ & $-0,14$ & 0,10 & 0,08 & $-0,37$ & 2,61 & 0,03 & 2,40 & 0,04 \\
\hline & & $(1,05)$ & $(0,94)$ & $(1,00)$ & $(0,90)$ & $(0,78)$ & $(1,25)$ & & & & \\
\hline \multirow{4}{*}{ Familiares } & \multirow{2}{*}{$\begin{array}{l}\text { Años de escolaridad } \\
\text { madre }\end{array}$} & 11,42 & 11,16 & 9,20 & 10,53 & 10,64 & 8,58 & 2,60 & 0,00 & 4,26 & 0,00 \\
\hline & & $(4,32)$ & $(3,33)$ & $(4,16)$ & $(3,34)$ & $(3,54)$ & $(4,85)$ & & & & \\
\hline & \multirow{2}{*}{$\begin{array}{l}\text { Años de escolaridad } \\
\text { padre }\end{array}$} & 12,54 & 10,51 & 10,81 & 10,15 & 10,53 & 8,26 & 0,80 & 0,55 & $6,64^{*}$ & 0,00 \\
\hline & & $(3,81)$ & $(3,88)$ & $(4,38)$ & $(3,35)$ & $(3,72)$ & $(4,13)$ & & & & \\
\hline \multirow{2}{*}{ Académicas } & \multirow{2}{*}{ Puntaje EXANI-II } & 1044,38 & 983,17 & 1017,41 & 1012,66 & 1008,43 & 985,07 & 1,21 & 0,31 & $2,93^{\star}$ & 0,01 \\
\hline & & $(93,59)$ & $(80,09)$ & $(94,23)$ & $(87,66)$ & $(109,80)$ & $(93,26)$ & & & & \\
\hline
\end{tabular}

Fuente: elaboración propia.

Nota: *diferencia estadísticamente significativa.

\subsection{Diferencias estadísticamente significativas por la ubicación de la EN: urbano y rural}

Las seis EN participantes en el estudio se ubican en los tres municipios más grandes de Baja California, como ya se mencionó, tres en la zona urbana y tres en la zona rural. Por lo tanto, se consideró pertinente indagar en la existencia de diferencias entre las poblaciones de aspirantes en función de la zona en la que se ubican las EN a las que pretenden ingresar. En este sentido, se conformaron dos grupos: 1) EN de zona urbana (EN1, EN4 y EN5) con 44\% de la población de aspirantes, y 2) EN de zona rural (EN2, EN3 y EN6) con 56\% de los participantes en el estudio.

Las variables que resultaron con una diferencia significativa entre los dos grupos comparados se presentan en la Tabla X. En este caso las cuatro variables analizadas cumplieron con el supuesto de homocedasticidad a través de la Prueba de Levene, por lo que el resultado del Estadístico t si puede ser tomado en cuenta.

Se encontró que los participantes de la zona urbana tuvieron puntajes más altos en todas las variables que resultaron con diferencia estadísticamente significativa. Al comparar el ISEC se obtuvo una diferencia de media de $-0,30$ puntos $(\mathrm{t}(343)=-2,82, \mathrm{p}<0,05)$, a favor de la zona urbana que tiene un ISEC más favorable $(\bar{X}=0,13, \mathrm{DE}=0,92)$. Las EN ubicadas en la zona urbana obtuvieron en promedio 
un índice positivo: EN1 0,23, EN4 0,10 y EN5 0,08; mientras que las ubicadas en la zona rural obtuvieron un puntaje negativo: EN2 -0,01, EN3 -0,14 y EN6 -0,37.

Estos cálculos permiten inferir que las condiciones socioeconómicas de las familias de los estudiantes de las tres EN ubicadas en zona urbana son un poco más favorables que el resto de las instituciones, en medida que se alejan del puntaje cero. Es importante contar con esta información, pues «el entorno cultural en el que se desarrollan los jóvenes puede llegar a favorecer o a entorpecer el desempeño educativo» (Guzmán y Serrano, 2007: 8).

En relación a las características familiares, cuya importancia se evidencia en otros estudios (Solís, 2015; Silva, 2014), se encontró diferencia en la escolaridad de ambos padres (madre t $(340)=-2,71$, $\mathrm{p}<0,05$ y padre t $(326)=-2,44, \mathrm{p}<0,05)$. En este rubro también la población urbana tuvo resultados mayores a la población rural al presentar los padres un año más de escolaridad, lo que los ubica en bachillerato inconcluso. En lo académico también se presentaron diferencias en el puntaje en el EXANI-II ( $(317)=-2,35, \mathrm{p}<0,05)$, en contra de la zona rural $(\bar{X}=996,39, \mathrm{DE}=90,27)$.

A partir de las características descritas, es posible afirmar que «en un sistema que pone a competir a todos por igual (...) los estudiantes menos dotados, tienen menores oportunidades y se encuentran en situación de desventaja frente a otros estudiantes) (Casillas et al., 2007: 13).

\section{Tabla X. Variables con diferencia significativa entre aspirantes por la zona en la que se ubica la EN}

\begin{tabular}{|c|c|c|c|c|c|c|c|c|}
\hline \multirow{4}{*}{ SECCIÓN } & \multirow{4}{*}{ VARIABLE } & & & \multicolumn{5}{|c|}{ RESULTADOS ESTADÍSTICOS } \\
\hline & & \multicolumn{2}{|c|}{ GRUPOS } & \multicolumn{2}{|c|}{$\begin{array}{c}\text { DIFERENCIA } \\
\text { DE VARIANZA }\end{array}$} & \multicolumn{3}{|c|}{$\begin{array}{l}\text { DIFERENCIA } \\
\text { DE MEDIAS }\end{array}$} \\
\hline & & $\begin{array}{c}\text { URBANO } \\
\text { MEDIA } \\
\text { (DESVIACIÓN } \\
\text { ESTÁNDAR) }\end{array}$ & $\begin{array}{c}\text { RURAL } \\
\text { MEDIA } \\
\text { (DESVIACIÓN } \\
\text { ESTÁNDAR) }\end{array}$ & $\begin{array}{l}\text { Estadístico } \\
\text { de Levene }\end{array}$ & Significancia & Estadístico T & $\begin{array}{l}\text { Diferencia } \\
\text { de media }\end{array}$ & Significancia \\
\hline & & $(\mathrm{N}=195)$ & $(\mathrm{N}=150)$ & & & & & \\
\hline \multirow{2}{*}{ Socio-económicas } & \multirow{2}{*}{ ISEC } & 0,13 & $-0,17$ & 3,03 & 0,08 & $-2,82^{\star}$ & $-0,30$ & 0,01 \\
\hline & & $(0,07)$ & $(1,08)$ & & & & & \\
\hline \multirow{4}{*}{ Familiares } & \multirow{2}{*}{$\begin{array}{l}\text { Años de escolaridad } \\
\text { madre }\end{array}$} & 10,81 & 9,64 & 1,69 & 0,19 & $-2,71^{\star}$ & $-1,17$ & 0,00 \\
\hline & & $(3,69)$ & $(4,28)$ & & & & & \\
\hline & \multirow{2}{*}{$\begin{array}{l}\text { Años de escolaridad } \\
\text { padre }\end{array}$} & 10,96 & 9,88 & 1,08 & 0,30 & $-2,44^{\star}$ & $-1,07$ & 0,02 \\
\hline & & $(3,71$ & $(4,26)$ & & & & & \\
\hline \multirow{2}{*}{ Académicas } & \multirow{2}{*}{ Puntaje EXANI-II } & 1021,08 & 996,39 & 0,78 & 0,38 & $-2,35^{\star}$ & $-24,69$ & 0,02 \\
\hline & & $(94,93)$ & $(90,27)$ & & & & & \\
\hline
\end{tabular}

Fuente: elaboración propia.

Nota: *diferencia estadísticamente significativa.

\section{Conclusiones}

Ante el avance educativo de las sociedades actuales la ES está en proceso de masificación, situación que abrió mayores oportunidades de acceso a este nivel educativo a más personas. Sin embargo, en sociedades como la mexicana «las instituciones de educación superior se han convertido en un filtro social y han desarrollado mecanismos para seleccionar a quienes ingresanı (Guzmán y Serrano, 2007), debido a que la oferta educativa en muchas ocasiones es menor a la demanda. Esta situación pone en entre dicho la garantía de igualdad en el servicio educativo de nivel superior, ya que continúan los casos en que las oportunidades de ingreso no son equivalentes para la totalidad de las personas interesadas en estudiar una carrera universitaria. 
De manera especial se destacan las brechas existentes en las regiones más vulnerables del país, ubicadas en su mayoría en las zonas rurales mexicanas. De tal suerte que es necesario enfatizar los esfuerzos para reducir las desigualdades sociales y fomentar el acceso equitativo a la educación superior (Jaimes et al., 2019), a través de la implementación de políticas públicas que permitan atender los rezagos en esta materia que han caracterizado al sistema educativo mexicano (Silva, 2014).

A partir de la revisión de literatura realizada en el estudio, los autores plantean la escasa producción académica acerca de expectativas y decisiones escolares de los estudiantes, al no ocupar un lugar central en la investigación social, al menos en Latinoamérica. En consecuencia, sostienen que es necesaria la producción de más estudios sobre las decisiones escolares, además de acercamientos cualitativos que permitan un análisis más minucioso y la obtención de información valiosa sobre los aspirantes.

Los autores afirman que «es fundamental realizar la descripción de estas trayectorias para que constituyan el punto de partida para realizar otros estudios que expliquen las causas o factores que la determinan y así emprender acciones para atenderlos» (Ochoa et al., 2017: 11). Además, se pretende que la investigación aporte los elementos necesarios para la construcción de políticas pertinentes enfocadas a la equidad social, las cuales hasta el momento se han concentrado en ampliar las oportunidades de acceso y la aplicación de medidas compensatorias, enfoque que ha resultado insuficiente (Silva, 2014; Solís, 2015).

Si bien la equidad no se considera una meta alcanzada en el ámbito general de la ES, menos lo es en el área específica de la formación inicial de docentes de educación básica. Por lo tanto, el tema del análisis de las características de los aspirantes como referente de las condiciones en las que ingresan a la EN no ha sido abordado a profundidad. En este sentido, este trabajo pretende contribuir al campo de conocimiento específico de la formación del profesorado al caracterizar a la población de aspirantes de una de las entidades federativas del país. Además de puntualizar las condiciones de desventaja en las que se presentan los aspirantes de las EN ubicadas en la zona rural del estado.

En consecuencia es fundamental identificar otras características de los aspirantes, además del puntaje en un examen de conocimiento como el EXANI-II, que permitan conocer con mayor profundidad a quienes pretenden formarse para ser el profesorado de primaria en México. Estas características pueden favorecer u obstaculizar el recorrido académico de los aspirantes, desde su ingreso hasta la conclusión óptima de sus estudios normalistas (Jaimes et al., 2019), por lo que su identificación sistemática es imperante (Reyes, 2017).

Este tipo de estudios no se realizaban anteriormente, pues «la cuestión de la caracterización y conocimiento de las condiciones socioeconómicas y culturales de los estudiantes era a un tiempo un asunto olvidado e ineludiblè (Ariño, 2014: 21). En cambio, ahora se reconoce la relevancia de contar con información que permita la definición de perfiles más completos de los estudiantes de ES y, o bien, en su etapa de candidatos.

Como se presentó en la sección de resultados, las características de los aspirantes corresponden a diferentes tipos tanto de orden personal como familiar y contextual. En general, dan muestra del bajo nivel educativo de las familias de donde provienen los participantes, situación coincidente en otras EN mexicanas (Reyes, 2017). Lo que permite afirmar que en su mayoría, los participantes serán la primera generación de estudiantes universitarios en sus familias y los posiciona negativamente al enfrentarse a las exigencias inherentes a la ES.

Además, destacan las diferencias encontradas entre las escuelas urbanas y rurales donde se mostró la situación de desigualdad en la que viven los aspirantes. Específicamente se enfatiza la situación vulne- 
rable de los aspirantes a ingresar a la EN6, quienes son desfavorecidos en todos los rubros estudiados. Estos resultados coinciden con otras investigaciones (Casillas et al., 2007; Guzmán y Serrano, 2007; Silva, 2014), que afirman que a pesar de contar con los requisitos de ingreso, las condiciones de vulnerabilidad de los aspirantes los colocan en franca desventaja frente a los otros grupos de aspirantes estudiados, lo que disminuye sus posibilidades de ingreso y culminación de sus estudios superiores, y posteriormente, su acceso al campo laboral.

Ante este panorama es imprescindible revisar que el proceso de selección de aspirantes a las EN mexicanas no sea un proceso inequitativo (Guzmán y Serrano, 2011) en donde la influencia de los factores socioeconómicos tenga un peso determinante. Por el contrario, que sea un medio para la recuperación información relevante para el diseño de políticas educativas, de carácter institucional, estatal y federal, que permitan el acompañamiento de los candidatos. Pues así, una vez que ingresen a la EN, será posible la implementación de programas de becas y tutorías individualizadas.

Aunque ya se han implementado programas compensatorios para las comunidades vulnerables, se considera que este acompañamiento puede apoyar la adecuada consecución de los estudios normalistas y el egreso de profesores de primaria con mejores perfiles académicos, que finalmente podrán impactar en su movilidad social (Herrera, 2019) y en el favorecer la equidad en las comunidades de donde provienen.

Con las evidencias presentadas en este artículo se busca abrir la discusión acerca del tema para hacerlo visible en el contexto mexicano y latinoamericano, para la posible generación de políticas educativas más contextualizadas, enfocadas a la equidad educativa. Esto para garantizar el ingreso y la atención efectiva de todos los jóvenes, específicamente aquellos provenientes de estratos sociales desfavorecidos (Silva, 2014), interesados en integrar el magisterio mexicano del futuro.

\section{Referencias bibliográficas}

Alonso, Carlos (2014): "Familia, escuela y clase social: sobre los efectos perversos de la implicación familiar". Revista de la Asociación de Sociología de la Educación, 7 (2), 395-409.

Alfonzo, Isabel; Cáceres, Teresa y Santana, Yaiza (2018): "Erasmus + Student Profile in the Development of Intercultural Competence: A Case Study". Revista Electrónica de Lingüistica Aplicada, 16 (1), 103-117.

Ariño, Antonio (2014): “La dimensión social en la educación superior”. Revista de la Asociación de Sociología de la Educación, 7 (1), 17-41.

Casillas, Miguel; Chaín, Ragueb y Jácome, Nancy (2007): “Origen social de los estudiantes y trayectorias estudiantiles en la Universidad Veracruzana”. Revista de la Educación Superior, 36 (2), 7-29.

Fortoul, Bertha; Güemes, Carmela; Martell, Flor y Reyes, Eugenia (2013): "Formación inicial de docentes para la Educación Básica” en Patricia Ducoing y Betha Fortoul (coords.). Procesos de formación. Vol. I. México: COMIE.

Gratacós, Gloria (2014). La motivación de los futuros profesores. Aula Magna 2.0 (en línea). http://cuedespyd.hypotheses.org/461\#content, consultado el 30 de noviembre de 2016.

Guzmán, Carlota y Serrano, Olga (2011): “Las puertas del ingreso a la educación superior: el caso del concurso de selección a la licenciatura de la UNAM”. Revista de la Educación Superior, 40 (1), 31-53. 
Hernández, Luis (2018): "Perfil sociodemográfico y académico en estudiantes universitarios respecto a su autoeficacia académica percibida". Psicogente, 21 (39), 35-49. https://doi.org/10.17081/psico.21.39.2820.

Herrera, Damián (2019): “¿Quién estudia en la universidad? La dimensión social de la universidad española en la segunda década del siglo XXI”. Revista de la Asociación de Sociología de la Educación, 12 (1), 7-23. https://doi.org/10.7203/RASE.12.1.13117.

Jaimes, Yadirah; Reynoso, Iris y Ferrer, Saúl (2019): “El proceso de selección, el perfil de ingreso a la Educación Normal y las características regionales: el caso de la Escuela Normal Rural Gral. Emiliano Zapata". Revista Interdisciplinaria de Estudios Latinoamericanos, 3 (2), 81-90.

Litalien, David; Gillet, Nicolas; Gagné, Maryléne; Ratelle, Catherine y Morin, Alexandre (2019): “Self-Determined Motivation Profiles among Undergraduate Students: A Robust Test of Profiles Similarity as a Function of Gender and Age". Learning and Individual Differences, 70, 39-52. https://doi. org/10.1016/j.lindif.2019.01.005.

Loubet-Orozco, Roxana (2018): “Contribución de la sociología en la formación docente. Un ejercicio de intervención en México". Revista de la Asociación de Sociología de la Educación, 11 (1), 5-20. https://doi. org/10.7203/RASE.11.1.10598.

Mudavanhu, Young (2015): “Contradictions and Tensions in Students' Motives of Enrolling in a Teacher Education Programme in Zimbabwe". Journal of Education and Training Studies, 3 (2), 159-170. https:// doi.org/10.11114/jets.v3i2.596.

Ochoa, Manuel; Vázquez, Mario y González, Isolina (2017): "Estudio descriptivo de las características de ingreso de los alumnos de la Licenciatura en Ciencias de la Educación del ITSON Unidad Obregón Cohorte 2014". Trabajo presentado en el XIV Congreso Nacional de Investigación Educativa. San Luis Potosí, México.

Reyes, Teresa (2017): "Caracterización del estudiante de nuevo ingreso a la Educación Normal. Generaciones: 2014 y 2015”. Trabajo presentado en el XIV Congreso Nacional de Investigación Educativa. San Luis Potosí, México.

Roloff, Janina; Klusmann, Uta; Lüdtke, Oliver y Trautwein, Ulrich (2015): “Who Becomes a Teacher? Challenging the 'Negative Selection' Hypothesis”. Learning and Instruction, (36), 46-56. https://doi. org/10.1016/j.learninstruc.2014.11.005.

Silva, Marisol (2014): "Equidad en la educación superior mexicana: el reto persistente". Universidades, 59, 23-25.

Smith, Emma y White, Patrick (2015): “What Makes a Successful Undergraduate? The Relationship Between Student Characteristics, Degree Subject and Academic Success at University". British Educational Research Journal, 41 (4), 686-708. https://doi.org/10.1002/berj.3158.

Solís, Patricio (2015). Desigualdad vertical y horizontal en las transiciones educativas en México. México: Centro de Estudios Espinosa Yglesias.

Spicker, Paul; Álvarez, Sonia y Gordon, David (2009). Pobreza: un glosario internacional. Buenos Aires: CLACSO. 
Tejuca, Mayra; Gutiérrez, Onil; Hernández, Gissell y García, Irina (2017): “Análisis del acceso a la educación superior cubana de los estudiantes de preuniversitario en el curso 2014-2015”. Revista Cubana Educación Superior, 1, 165-186.

Yáñez, Adrián; Vera, José y Mungarro, Enrique (2014): “El proceso de admisión de las escuelas normales y los antecedentes socioeconómicos como predictores del rendimiento académico". Revista Intercontinental de Psicología y Educación, 16 (2), 111-129.

\section{Notas biográficas}

Jihan García-Poyato Falcón es Licenciada en Educación Primaria por la «Benemérita Escuela Normal Estatal, Profesor Jesús Prado Luna» (BENEPJPL) y Licenciada en Sociología por la Universidad Autónoma de Baja California (UABC); Maestra en Ciencias Educativas por el Instituto de Investigación y Desarrollo Educativo (IIDE-UABC) y actualmente es estudiante del último cuatrimestre del Doctorado en Ciencias Educativas en el mismo instituto. Cuenta con una Especialidad en Herramientas Básicas para la Investigación Educativa por la Universidad Autónoma del Estado de Morelos. Docente de la Licenciatura en Educación Primaria desde 2004 y fue Coordinadora del Departamento de Investigación de 2012 a 2016 en la BENEPJPL. Docente en la Licenciatura en Ciencias de la Educación en UABC de 2009 a 2016.

Graciela Cordero Arroyo es Licenciada en Pedagogía por la Universidad Nacional Autónoma de México (UNAM), maestra en Educación por la Universidad de Harvard y doctora en Filosofía y Ciencias de la Educación por la Universidad de Barcelona. Investigadora Titular del Instituto de Investigación y Desarrollo Educativo (IIDE) de la Universidad Autónoma de Baja California (UABC). Es miembro del Sistema Nacional de Investigadores desde 1999. Editora fundadora de la Revista Electrónica de Investigación Educativa (REDIE) de 1999 a 2003. Fue directora del IIDE-UABC de 2003 a 2011. Recientemente ha sido nombrada presidenta del Observatorio Internacional de la Profesión Docente que gestiona la Universidad de Barcelona. 